\title{
Dynamic Stabilization of the Optical Resonances of Single Nitrogen-Vacancy Centers in Diamond
}

\author{
V. M. Acosta, ${ }^{1, *}$ C. Santori, ${ }^{1}$ A. Faraon,,${ }^{1}$ Z. Huang, ${ }^{1}$ K.-M. C. Fu, ${ }^{1,2}$ A. Stacey, ${ }^{3}$ D. A. Simpson, ${ }^{3}$ K. Ganesan, ${ }^{3}$ \\ S. Tomljenovic-Hanic, ${ }^{3}$ A. D. Greentree, ${ }^{3,4}$ S. Prawer, ${ }^{3}$ and R. G. Beausoleil ${ }^{1}$ \\ ${ }^{1}$ Hewlett-Packard Laboratories, 1501 Page Mill Road, Palo Alto, California 94304, USA \\ ${ }^{2}$ Departments of Physics and Electrical Engineering, University of Washington, Seattle, Washington 98195, USA \\ ${ }^{3}$ School of Physics, University of Melbourne, Melbourne VIC 3010, Australia \\ ${ }^{4}$ Applied Physics, School of Applied Sciences, RMIT University, Melbourne 3001, Australia
}

(Received 22 December 2011; published 14 May 2012)

\begin{abstract}
We report electrical tuning by the Stark effect of the excited-state structure of single nitrogen-vacancy (NV) centers located $\lesssim 100 \mathrm{~nm}$ from the diamond surface. The zero-phonon line (ZPL) emission frequency is controllably varied over a range of $300 \mathrm{GHz}$. Using high-resolution emission spectroscopy, we observe electrical tuning of the strengths of both cycling and spin-altering transitions. Under resonant excitation, we apply dynamic feedback to stabilize the ZPL frequency. The transition is locked over several minutes and drifts of the peak position on timescales $\gtrsim 100 \mathrm{~ms}$ are reduced to a fraction of the single-scan linewidth, with standard deviation as low as $16 \mathrm{MHz}$ (obtained for an NV in bulk, ultrapure diamond). These techniques should improve the entanglement success probability in quantum communications protocols.
\end{abstract}

DOI: 10.1103/PhysRevLett.108.206401

Integrated photonic networks based on cavity-coupled solid-state spin impurities offer a promising platform for scalable quantum computing [1-5]. A key ingredient for this technology is the generation and interference of indistinguishable photons emitted by pairs of identical spin qubits [6-8]. This requires spectrally stable emitters with identical level structure, a formidable challenge in the solid-state environment.

A potential solution is to use external control to counteract sample inhomogeneities. In candidate systems based on single molecules [9-11], quantum dots [12,13], and negatively-charged nitrogen-vacancy (NV) centers in diamond [14-16], the level structure can be statically tuned via the dc Stark effect. However, the spectral stability of emitters in these systems is often hampered by local fluctuations which cause the emission frequency to change with time, a phenomenon known as spectral diffusion [17]. Previous attempts to address this problem have focused on improving the host material [14,18-20] or using post-selection techniques [16,21-23], but a robust, high-yield solution is still lacking.

The diamond NV center is an attractive spin qubit, as it exhibits a unique combination of long-lived spin coherence [24] and efficient optical control and readout [25,26]. However integration into on-chip photonic networks requires NV centers to be located near nanostructured surfaces,

Published by the American Physical Society under the terms of the Creative Commons Attribution 3.0 License. Further distribution of this work must maintain attribution to the author(s) and the published article's title, journal citation, and DOI.
PACS numbers: 71.55.Cn, 71.70.Ej, 78.55.- m, 81.05.ug

where inhomogeneous strain and spectral diffusion can be particularly problematic [27,28]. In this Letter, we first demonstrate electrical control over the zero-phonon line (ZPL) transition frequencies, as well as probabilities for both cycling and $\Lambda$-type transitions, of single NV centers located near the diamond surface. We then show that spectral diffusion of the ZPL can be suppressed to $16 \mathrm{MHz}$ standard deviation, on time scales $\gtrsim 100 \mathrm{~ms}$, by providing rapid electrical feedback to compensate for local field fluctuations.

The negatively charged nitrogen-vacancy (NV) center has $C_{3 v}$ symmetry, and the basic energy structure is depicted in Fig. 1(a). The spin-triplet ground state, ${ }^{3} A_{2}$, is split such that the $m_{s}=0$ spin projection (labeled $|0\rangle$ throughout) is separated from the degenerate $m_{s}= \pm 1$ levels $(| \pm 1\rangle)$ by $D_{\mathrm{GS}}=2.88 \mathrm{GHz}$ at $T \lesssim 100 \mathrm{~K}$ [29]. The optically excited state (ES) is a spin triplet and orbital doublet with ${ }^{3} E$ symmetry, and its fine structure has been studied theoretically in detail $[30,31]$. The Hamiltonian describing the ES manifold is

$$
\mathcal{H}_{\mathrm{ES}}=H_{\mathrm{SO}}+\mathcal{H}_{\mathrm{SS}}+\mathcal{H}_{\mathrm{Stark}}, \mathcal{H}_{\text {Stark }}=-\vec{d} \cdot \vec{F},
$$

where $\mathcal{H}_{\mathrm{SO}}, \mathcal{H}_{\mathrm{SS}}$, and $\mathcal{H}_{\text {Stark }}$ contain, respectively, the spin-orbit, spin-spin, and Stark effect contributions; $\vec{d}$ is the electric dipole moment, and $\vec{F}$ is the electric field. The effect of a strain is treated as an effective electric field $[32,33]$.

We first consider the influence of $\mathcal{H}_{\text {Stark }}$ on only the orbital portion of the ES wave function, consisting of two eigenstates, $\left.\left\{\left|\mathcal{E}_{x}\right\rangle, \mid \mathcal{E}_{y}\right\}\right\rangle$, initially degenerate at zero field. Under electric fields, the orbitals exhibit energy shifts, $\Delta(\vec{F})$, of

$$
\Delta_{\mathcal{E}_{x}}(\vec{F})=d_{\|} F_{\|}+d_{\perp} F_{\perp}, \quad \Delta_{\mathcal{E}_{y}}(\vec{F})=d_{\|} F_{\|}-d_{\perp} F_{\perp},
$$



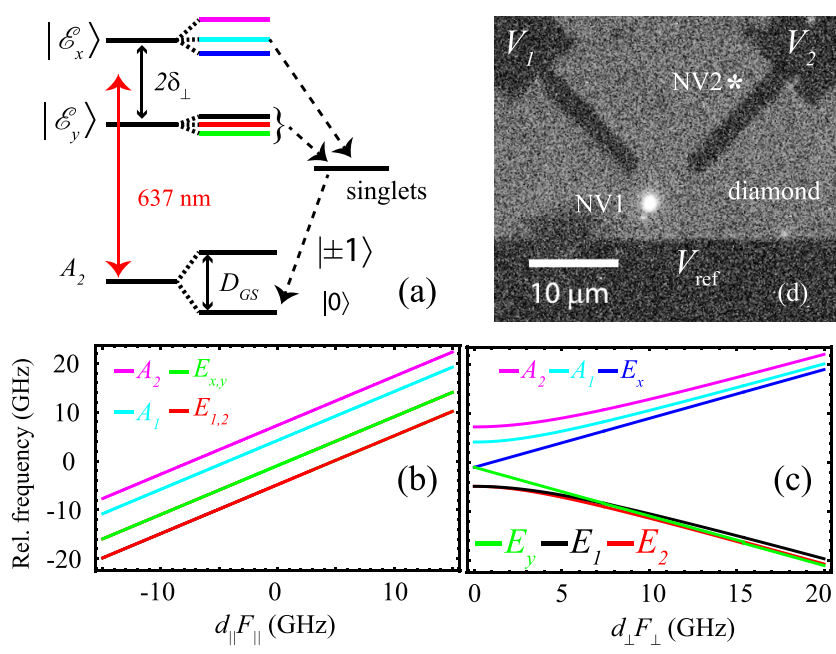

FIG. 1 (color online). (a) NV level structure at high transverse field $\left(d_{\perp} F_{\perp} \equiv \delta_{\perp}>15 \mathrm{GHz}\right.$ ). Dashed lines indicate the spinselective decay path responsible for optical pumping. (b) Effect of longitudinal and (c) transverse electric fields on the excited state levels. (d) Fluorescence micrograph of the electrode structure. NV1 was illuminated by $532 \mathrm{~nm}$ light and appears white, while the position of NV2 is denoted with an asterisk. Metal electrodes appear as shadows.

where the directions are with respect to the NV symmetry axis. Longitudinal fields do not lift the orbital degeneracy and result only in equal, linear shifts of all levels. Transverse fields split the orbitals into two branches with an energy difference, $2 \delta_{\perp} \equiv\left(\Delta_{\mathcal{E}_{x}}-\Delta_{\mathcal{E}_{y}}\right)$, that grows linearly with increasing field. The spacings between groundstate sublevels remain relatively unaffected by electric fields $[34,35]$. The ground state may have a longitudinal dipole moment, $d_{\mathrm{GS}, \|}[30,31]$, but in experiments we only resolved $\Delta d_{\|} \equiv d_{\|}-d_{\mathrm{GS}, \|}$.

Incorporating spin interactions results in a set of six eigenstates, $\left\{\left|A_{2}\right\rangle,\left|A_{1}\right\rangle,\left|E_{x}\right\rangle,\left|E_{y}\right\rangle,\left|E_{1}\right\rangle,\left|E_{2}\right\rangle\right\}$, ordered from highest to lowest energy (at low field). Figures 1(b) and 1(c) show the effect of $\mathcal{H}_{\mathrm{ES}}$ on all six ES energies due to, respectively, $F_{\|}$and $F_{\perp}$.

We focused most of our study on NV centers close to the diamond surface, a necessary feature for future integration with nanophotonic devices. Our sample, described in detail elsewhere [36,37], consists of a high-purity single-crystal, [100]-oriented diamond substrate with a $\sim 100 \mathrm{~nm}$ thick chemical-vapor-deposition-grown layer with $[\mathrm{NV}] \approx$ $10^{6} \mathrm{~cm}^{-2}$. The two NV centers studied in this work, labeled NV1 and NV2, are located in this surface layer [37]. Lithographically defined metal electrodes [15] were deposited on the surface [Fig. 1(d)]. The layout of the electrodes (labeled $V_{1}, V_{2}$, and $V_{\text {ref }}$ ) permits tuning of electric fields in any in-plane direction near the center of the structure [see Supplementary Information (SI) [38]].

A confocal microscope was used to excite and collect emitted light from a diamond sample in thermal contact with the cold finger of a flow-through, liquid-helium cryostat.
The cold finger was maintained at a temperature $T \approx 7 \mathrm{~K}$, and no magnetic field was applied. Two forms of spectroscopy were employed: high-resolution emission spectroscopy and photoluminescence excitation spectroscopy.

For emission spectroscopy, $2 \mathrm{~mW}$ of $532 \mathrm{~nm}$ laser light was focused by a 0.6-numerical-aperture objective onto NV1, exciting through the phonon sideband (PSB) near saturation. The collected emission was spectrally filtered to direct ZPL light (636-638 nm) to a high-resolution grating spectrometer. The optical polarization was chosen to ensure excitation of both orbital branches [39]. Figure 2(a) shows
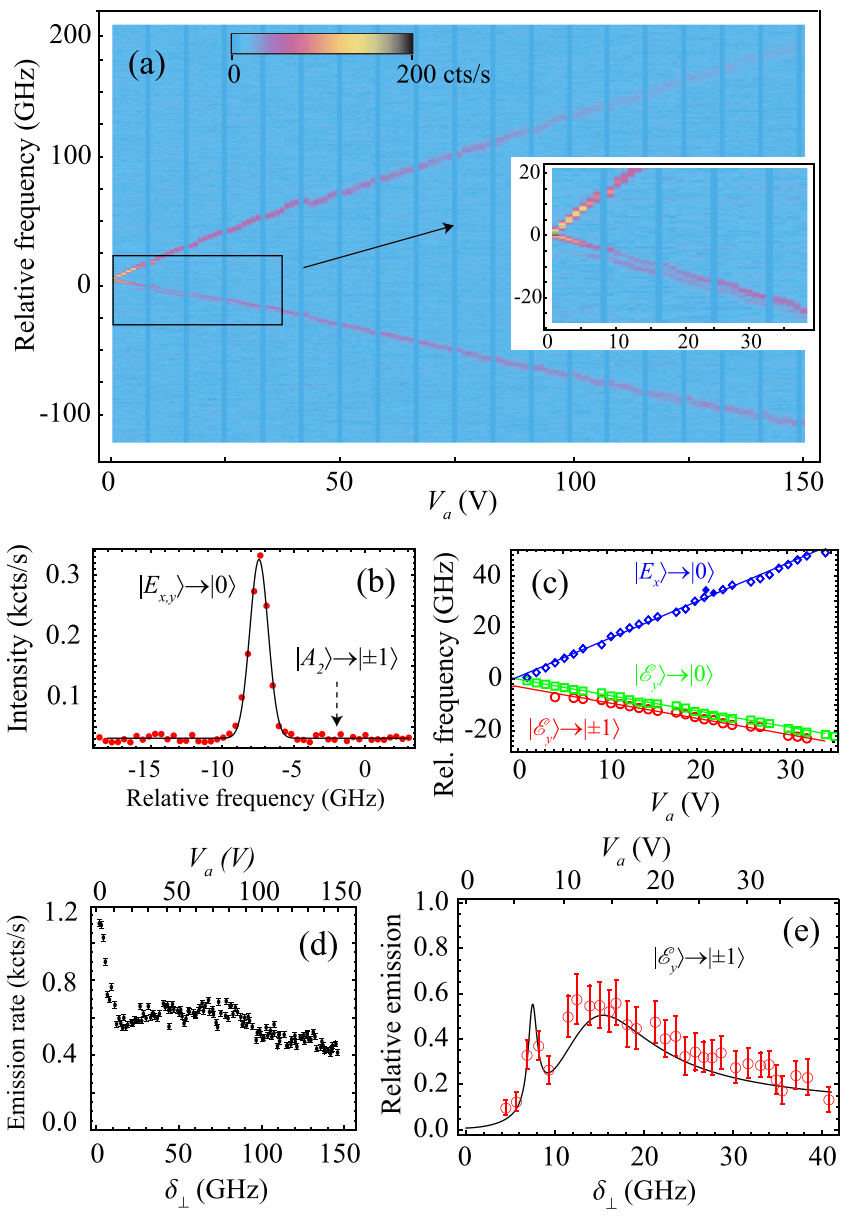

FIG. 2 (color online). (a) Stark emission spectroscopy. $V_{a}$ was slowly scanned (1 V/min) and emission spectra were obtained in one-minute intervals. A dark exposure frame was taken every 8th frame (cyan vertical stripes). Emission frequencies are relative to $470.45 \mathrm{THz}(637.25 \mathrm{~nm})$. Low-field data are inset. (b) Emission spectrum for $V_{a}=1 \mathrm{~V}$ with expected peak positions labeled. The $\left|E_{x, y}\right\rangle \rightarrow|0\rangle$ emission line is fit with a Gaussian profile. (c) Low-field peak positions from (a) and global fit based on Eq. (1). Lorentzian fit uncertainty is smaller than the plotted symbols. (d) Total ZPL emission versus $\delta_{\perp}$. The emission rate was calculated by subtracting the mean background and summing counts over a range of $\sim 3$ FWHM linewidths centered at each peak. Error bars are based on Poissonian noise. (e) Relative intensity of the $\left|\mathcal{E}_{y}\right\rangle \rightarrow| \pm 1\rangle$ emission line along with fit (see text). 
the emission spectra versus voltage, $V_{a}$, applied simultaneously on $V_{1}$ and $V_{\text {ref }}$, with $V_{2}=0$. By varying $V_{a}$ from 0 to $150 \mathrm{~V}$, we observe linear tuning of emission lines over a range exceeding $300 \mathrm{GHz}$. Such a wide tuning range, enabled by the enhanced fields provided by our devices (SI [38]), is essential to compensate for the large intrinsic fields typical in nanophotonic devices [28].

Depending on the applied voltage, we resolve between one and three emission lines. At $V_{a} \approx 1 \mathrm{~V}$ we observe a single emission line [Fig. 2(b)] with full-width-at-halfmaximum (FWHM) linewidth of $1.4(2) \mathrm{GHz}$, near the spectrometer resolution of $\sim 0.9 \mathrm{GHz}$. We interpret this peak as containing unresolved contributions from the $\left|E_{x, y}\right\rangle \rightarrow|0\rangle$ cycling transitions [40,41]. Taking into consideration the absence of other peaks, in particular, the $\left|A_{2}\right\rangle \rightarrow| \pm 1\rangle$ cycling transition [23], and the observed noise floor, we place a bound on the ground-state spin polarization $\mathcal{P}_{\mathrm{GS}} \equiv P_{0} /\left(P_{0}+P_{ \pm 1}\right) \gtrsim 90 \%$, where $P_{i}$ is the occupation probability of state $|i\rangle$.

Upon application of transverse fields, the spin character of the levels in the upper $\left|\mathcal{E}_{x}\right\rangle$ orbital branch, $\left\{\left|A_{2}\right\rangle\right.$, $\left.\left|A_{1}\right\rangle,\left|E_{x}\right\rangle\right\}$, remain relatively unperturbed. In contrast, the spin character of levels in $\left|\mathcal{E}_{y}\right\rangle,\left\{\left|E_{y}\right\rangle,\left|E_{1}\right\rangle,\left|E_{2}\right\rangle\right\}$, mix at avoided crossings due to spin-spin interaction [30,31,40,42], making these levels useful for spin-altering $\Lambda$ schemes.

In the range $5 \mathrm{~V} \lesssim V_{a} \lesssim 35 \mathrm{~V}$, three emission lines are visible [inset of Fig. 2(a)]. Based on the positive, linear tuning, the upper peak is identified as $\left|E_{x}\right\rangle \rightarrow|0\rangle$. Lorentzian fits to these spectra reveal that the two lowest lines are on average separated by $2.7(2) \mathrm{GHz}$, which is comparable to $D_{\mathrm{GS}}$. Considering also the negative, linear tuning, we conclude that these peaks arise from $\left|\mathcal{E}_{y}\right\rangle \rightarrow|0\rangle$ and $\left|\mathcal{E}_{y}\right\rangle \rightarrow| \pm 1\rangle$ emission (the three levels within $\left|\mathcal{E}_{y}\right\rangle$ are nearly degenerate and unresolved here). The presence of these lines was previously predicted based on observations of spin-altering $\Lambda$-type transitions involving the lower orbital branch $[40,42,43]$. Figure 2(c) plots the emission frequencies along with a fit using a model based on Eq. (1), showing excellent agreement. The fitted parameters are $\Delta d_{\|} F_{\|} / V_{a}=0.42(2) \mathrm{GHz} / \mathrm{V}$ and $d_{\perp} F_{\perp} / V_{a}=$ $1.03(3) \mathrm{GHz} / \mathrm{V}$.

Even with significant emission to $| \pm 1\rangle$, we still do not observe $\left|A_{2}\right\rangle \rightarrow| \pm 1\rangle$ emission. Throughout, we find $\mathcal{P}_{\mathrm{GS}} \gtrsim 85 \%$. A likely explanation is that any population in $| \pm 1\rangle$ is quickly transferred to the metastable singlet levels [44], preventing the detection of $m_{s}= \pm 1$ emission lines. This is consistent with Fig. 2(d), where the total ZPL emission rate integrated over all lines is plotted as a function of one half the orbital splitting, $\delta_{\perp}$. Between $3 \lesssim$ $\delta_{\perp} \lesssim 10 \mathrm{GHz}\left(3 \lesssim V_{a} \lesssim 10 \mathrm{~V}\right)$, the emission rate falls precipitously before leveling off at less than half the initial rate.

The relative intensity of the emission lines gives further insight into the ES properties. Figure 2(e) plots the intensity of the $\left|\mathcal{E}_{y}\right\rangle \rightarrow| \pm 1\rangle$ emission line, normalized by the total emission from $\left|\mathcal{E}_{y}\right\rangle$, as a function of $\delta_{\perp}$. Evidently, the applied field is a powerful knob in tuning the relative transition strengths in this $\Lambda$ system. Two peaks for the emission of $\left|\mathcal{E}_{y}\right\rangle \rightarrow| \pm 1\rangle$ are present at $\delta_{\perp} \approx 7$ and $15 \mathrm{GHz}$. These features correspond to level anticrossings [see Fig. 1(c)], where maximal mixing of levels in the lower orbital branch occurs. The degree of mixing depends sensitively on both the magnitude of the transverse electric field and its angle, $\theta_{r}$, with respect to the $C_{3 v}$ reflection planes [40]. We model the relative emission intensity by assuming the NV center is excited from $|0\rangle$ to one of the three levels in the lower branch, $\left|\mathcal{E}_{y, i}\right\rangle$. The probability that emission is back to $| \pm 1\rangle$ is then $\sum_{i} P_{0, i}\left(\theta_{r}, \delta_{\perp}\right)$ $\left(1-P_{0, i}\left(\theta_{r}, \delta_{\perp}\right)\right)$, where $P_{0, i}\left(\theta_{r}, \delta_{\perp}\right)$ is calculated by taking the overlap of $\left|\mathcal{E}_{y, i}\right\rangle$ with $m_{s}=0$ and tracing over orbital degrees of freedom. Here we assume all levels in $\left|\mathcal{E}_{y}\right\rangle$ couple equally to the singlets. Using the model based on Eq. (1), we fit this formula to the data and find good agreement for $\theta_{r}=15(5)^{\circ}$.

Finally, we also observe a strong dependence of the relative emission between the upper and lower branches on $\delta_{\perp}$ [Fig. 2(a)]. Given the low temperature $T=7 \mathrm{~K}$, this may be due to a single-phonon orbital relaxation process. Phonon decay to the lower branch could contribute to the decreased total emission in Fig. 2(d). A detailed study will be the focus of future work. All of the effects described above were reproduced in subsequent voltage scans; see SI [38].

To realize even higher spectral resolution, we performed photoluminescence excitation (PLE) spectroscopy. Attenuated light $(\sim 60 \mathrm{nW})$ from a tunable, external-cavity diode laser $(-637 \mathrm{~nm})$ was used for ZPL excitation near saturation, and the collected light was filtered to direct PSB emission (650-800 $\mathrm{nm}$ ) to a single-photon-counting detector. Microwaves resonant with the ground-state spin transition, $D_{\mathrm{GS}}=2.877 \mathrm{GHz}$, were continuously applied to counteract optical pumping [40,45], and light from a repump laser $(532 \mathrm{~nm})$ was occasionally employed to reverse photoionization $[43,46,47]$.

Figure 3(a) plots PLE spectra for NV1 as a function of $V_{a}$, applied simultaneously to $V_{\text {ref }}$ and $V_{1}$, with $V_{2}=0$. Several excitation lines are resolved due to the presence of resonant microwave excitation. We fit the five strongest lines with Lorentzian profiles. The extracted peak positions are plotted in Fig. 3(b) along with a global fit to the model based on Eq. (1), yielding Stark coefficients $\Delta d_{\|} F_{\|} / V_{a}=$ $0.11(1) \mathrm{GHz} / \mathrm{V}$ and $d_{\perp} F_{\perp} / V_{a}=0.26(2) \mathrm{GHz} / \mathrm{V}$. These coefficients are about 4 times smaller than those realized under strong $532 \mathrm{~nm}$ excitation, consistent with recent observations of enhanced electrical tuning due to photoionization $[15,16]$.

We note that the average linewidth for single scans [39] was $\Gamma_{\mathrm{ss}}=0.14(3) \mathrm{GHz}$ for NV1 and $\Gamma_{\mathrm{ss}}=0.48(8) \mathrm{GHz}$ for NV2. In both cases, $\Gamma_{\mathrm{ss}}$ is much broader than the natural linewidth, $\Gamma_{\text {nat }} \approx 13 \mathrm{MHz}$, and is independent of scan rate up to $\sim 20 \mathrm{GHz} / \mathrm{s}$. This property requires further 

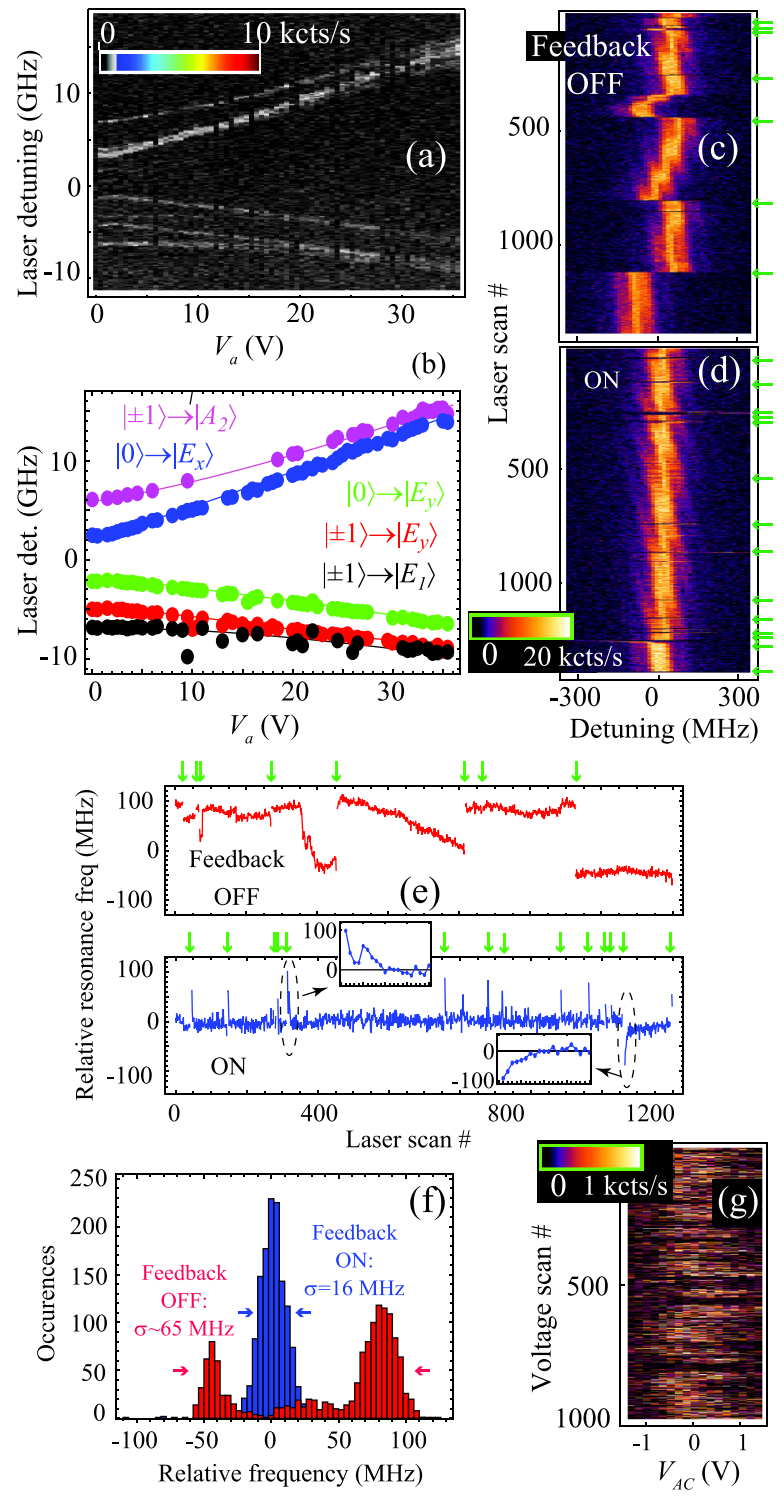

FIG. 3 (color online). (a) PLE spectra versus applied voltage for NV1. A $532 \mathrm{~nm}$ repump pulse $(2.5 \mu \mathrm{W}, 1 \mathrm{~s}$ duration) was applied every 60 s. (b) Fitted transition frequencies from (a) and global fit based on Eq. (1), with transitions labeled. (c) PLE spectra for a single NV in the Ural sample $\left(|0\rangle \rightarrow\left|E_{x}\right\rangle\right)$ at $V_{a}=$ $-4 \mathrm{~V}$. Green arrows indicate when the repump was applied. (d) PLE spectra for the same NV with voltage feedback applied.

(e) Fitted peak positions for scans in (c) [upper panel] and (d) [lower panel]. The recovery under feedback after two separate repump pulses are inset. (f) Histogram of the peak positions in (e). (g) $100 \mathrm{~s}$ segment of PLE spectra on NV2 obtained by rapidly scanning a voltage, $V_{\mathrm{ac}}$, with feedback applied to $V_{\mathrm{dc}}$.

investigation, as $\Gamma_{\mathrm{ss}} \approx \Gamma_{\text {nat }}$ has been observed elsewhere $[14,16,39]$.

A likely cause for NV spectral diffusion is charge dynamics due to photoionization of nearby defects. To investigate, we use PLE spectroscopy in a different device on the $|0\rangle \rightarrow\left|E_{x}\right\rangle$ transition of a single NV center in natural, type IIa (Ural) diamond. This sample was chosen due to the much narrower linewidth, $\Gamma_{\mathrm{ss}}=60(7) \mathrm{MHz}$, even after $\sim 15 \mathrm{MHz}$ of power broadening. Figure 3(c) shows typical PLE spectra for $200 \mathrm{~ms}$ scans with repump pulse $(\sim 10 \mu \mathrm{W}, 20 \mathrm{~ms}$ duration) applied only after the NV center had photoionized. The transition frequency drifts over a range significantly larger than $\Gamma_{\mathrm{ss}}$ during the $280 \mathrm{~s}$ data set.

Our solution to the spectral-drift problem is to actively adjust $V_{a}$ to compensate for the changing local field. We start with $V_{a}=-4 \mathrm{~V}$, and, during the back-scan of subsequent scans (final 10\% of each cycle), we employ software-controlled feedback with the following algorithm. We first determine the position and intensity of the peak fluorescence. If the intensity falls below a threshold, we apply a repump pulse and do not change $V_{a}$. Otherwise, we change $V_{a}$ based on optimized proportionality and integration inputs (see SI [38]).

Figure 3(d) shows PLE spectra under similar conditions as Fig. 3(c) but now with feedback applied. While $\Gamma_{\mathrm{ss}}$ remains unchanged, the center-frequency drift is substantially reduced. We fit the spectra in Figs. 3(c) and 3(d) with Lorentzian profiles and plot the extracted peak positions in Fig. 3(e). In the case of no feedback, two mechanisms of spectral drift are identified: large instantaneous jumps following application of the repump and slower drift in between repump pulses. Under feedback, spectral jumps still accompany repump pulses, but these are quickly compensated for. Two spectral jumps with the slowest recovery, 5-10 scans, are shown as insets. A figure of merit for the total drift is obtained by plotting the histogram of fitted peak positions from all PLE scans and determining the resulting standard deviation, $\sigma$ [Fig. 3(f)]. Without feedback, we find a nonuniform profile with $\sigma \approx 65 \mathrm{MHz}$. Under feedback, $\sigma=16 \mathrm{MHz}$, which is smaller than $\Gamma_{\mathrm{ss}}$ and comparable to $\Gamma_{\text {nat }}$.

This feedback technique can be applied at significantly higher bandwidth (here, up to $20 \mathrm{~Hz}$ scan repetition rate) without compromising stability. Throughout, we find that feedback reduces $\sigma$ to a fraction of $\Gamma_{\text {ss }}$. Similar results were obtained for NV1 and NV2 [Supplemental Information [38]] as well as for stabilizing the $| \pm 1\rangle \rightarrow A_{2}$ transition.

It is often advantageous to perform experiments with the excitation laser frequency fixed to an external reference. In this case, voltage feedback can still be employed by sweeping the ZPL transition frequency using an ac voltage, $V_{\mathrm{ac}}$, and providing stabilizing feedback to the dc component, $V_{\mathrm{dc}}$. With this technique, feedback can be applied continuously without substantially degrading photon indistinguishability, provided that the modulation depth and laser linewidth are sufficiently small.

Figure $3(\mathrm{~g})$ shows results of locking the NV2 $|0\rangle \rightarrow\left|E_{x}\right\rangle$ transition frequency using only applied voltages. We perform PLE spectroscopy as before except, instead of scanning the laser frequency, we ramp the voltage, $V_{\text {ac }}$ (applied to $V_{2}$ and $V_{\text {ref }}$ ), with amplitude $3 V_{p p}$ and period $0.1 \mathrm{~s}$. 
Meanwhile, $V_{\mathrm{dc}}$ is fed back to $V_{1}$, initially starting at $-8 \mathrm{~V}$, but varying by $\sim \pm 4 \mathrm{~V}$ throughout the $600 \mathrm{~s}$ measurement. After background subtraction, we collect on average $144 \mathrm{cts} / \mathrm{s}$. This compares favorably to the $34 \mathrm{cts} / \mathrm{s}$ collected without feedback (with repump applied every scan). The overall count rate can be further increased with improved collection efficiency $[48,49]$ and resonant Purcell enhancement $[28,50]$.

In summary, we have used the Stark effect to electrically tune and stabilize the structure of the NV center's excited state. Applied simultaneously to a pair of NV centers, these techniques pave the way for increased two-photon interference visibilities $[11,13,16]$ and heralded entanglement success probabilities [8].

We acknowledge support by the Defense Advanced Research Projects Agency (Award No. HR0011-09-10006), the Regents of the University of California, and the Australian Research Council (ARC) (Project No. LP100100524, DP1096288, and No. DP0880466). We thank T. Karle, B. Gibson, T. Ishikawa, B. Buckley, and A. Falk for valuable discussions.

*victor.acosta@hp.com

[1] J. L. O'Brien, Science 318, 1567 (2007).

[2] A. M. Stephens, Z. W. E. Evans, S. J. Devitt, A. D. Greentree, A. G. Fowler, W. J. Munro, J. L. O'Brien, K. Nemoto, and L. C. L. Hollenberg, Phys. Rev. A 78, 032318 (2008).

[3] S. Benjamin, B. Lovett, and J. Smith, Laser Photon. Rev. 3, 556 (2009).

[4] T. D. Ladd, F. Jelezko, R. Laflamme, Y. Nakamura, C. Monroe, and J. L. O’Brien, Nature (London) 464, 45 (2010).

[5] C. Santori, P.E. Barclay, K.-M. C. Fu, R. G. Beausoleil, S. Spillane, and M. Fisch, Nanotechnology 21, 274008 (2010).

[6] C. Cabrillo, J. I. Cirac, P. García-Fernández, and P. Zoller, Phys. Rev. A 59, 1025 (1999).

[7] L. I. Childress, J.M. Taylor, A. Sørensen, and M. D. Lukin, Phys. Rev. A 72, 052330 (2005).

[8] D. L. Moehring, P. Maunz, S. Olmschenk, K. C. Younge, D. N. Matsukevich, L.-M. Duan, and C. Monroe, Nature (London) 449, 68 (2007).

[9] M. Orrit, J. Bernard, A. Zumbusch, and R. Personov, Chem. Phys. Lett. 196, 595 (1992).

[10] U. P. Wild, F. Güttler, M. Pirotta, and A. Renn, Chem. Phys. Lett. 193, 451 (1992).

[11] R. Lettow, Y.L. A. Rezus, A. Renn, G. Zumofen, E. Ikonen, S. Götzinger, and V. Sandoghdar, Phys. Rev. Lett. 104, 123605 (2010).

[12] S. A. Empedocles and M. G. Bawendi, Science 278, 2114 (1997).

[13] R. B. Patel, A. J. Bennett, I. Farrer, C. A. Nicoll, D. A. Ritchie, and A. J. Shields, Nature Photon. 4, 632 (2010).

[14] P. Tamarat, T. Gaebel, J. R. Rabeau, M. Khan, A. D. Greentree, H. Wilson, L. C. L. Hollenberg, S. Prawer, P. Hemmer, F. Jelezko, and J. Wrachtrup, Phys. Rev. Lett. 97, 083002 (2006).

[15] L. Bassett, F. Heremans, C. Yale, B. Buckley, and D. Awschalom, Phys. Rev. Lett. 107, 266403 (2011).
[16] H. Bernien, L. Childress, L. Robledo, M. Markham, D. Twitchen, and R. Hanson, Phys. Rev. Lett. 108, 043604 (2012).

[17] W. P. Ambrose and W.E. Moerner, Nature (London) 349, 225 (1991).

[18] F. Jelezko, B. Lounis, and M. Orrit, J. Chem. Phys. 107, 1692 (1997).

[19] C. Santori, D. Fattal, J. Vučković, G. S. Solomon, and Y. Yamamoto, Nature (London) 419, 594 (2002).

[20] A. D. Greentree, P. Olivero, M. Draganski, E. Trajkov, J. R. Rabeau, P. Reichart, B. C. Gibson, S. Rubanov, S. T. Huntington, D. N. Jamieson, and S. Prawer, J. Phys. Condens. Matter 18, S825 (2006).

[21] S. Ates, S.M. Ulrich, S. Reitzenstein, A. Löffler, A. Forchel, and P. Michler, Phys. Rev. Lett. 103, 167402 (2009).

[22] L. Robledo, H. Bernien, I. van Weperen, and R. Hanson, Phys. Rev. Lett. 105, 177403 (2010).

[23] E. Togan, Y. Chu, A.S. Trifonov, L. Jiang, J. Maze, L. Childress, M. V. G. Dutt, A. S. Sorensen, P. R. Hemmer, A. S. Zibrov, and M. D. Lukin, Nature (London) 466, 730 (2010).

[24] G. Balasubramanian, P. Neumann, D. Twitchen, M. Markham, R. Kolesov, N. Mizuochi, J. Isoya, J. Achard, J. Beck, J. Tissler, V. Jacques, P. R. Hemmer, F. Jelezko, and J. Wrachtrup, Nature Mater. 8, 383 (2009).

[25] B. B. Buckley, G. D. Fuchs, L.C. Bassett, and D. D. Awschalom, Science 330, 1212 (2010).

[26] L. Robledo, L. Childress, H. Bernien, B. Hensen, P. F. A. Alkemade, and R. Hanson, Nature (London) 477, 574 (2011).

[27] K. M. C. Fu, C. Santori, P.E. Barclay, and R. G. Beausoleil, Appl. Phys. Lett. 96, 121903 (2010).

[28] A. Faraon, P. E. Barclay, C. Santori, K.-M. C. Fu, and R. G. Beausoleil, Nature Photon. 5, 301 (2011).

[29] V. M. Acosta, E. Bauch, M. P. Ledbetter, A. Waxman, L. S. Bouchard, and D. Budker, Phys. Rev. Lett. 104, 70801 (2010).

[30] M. W. Doherty, N. B. Manson, P. Delaney, and L. C. L. Hollenberg, New J. Phys. 13, 025019 (2011).

[31] J. Maze, A. Gali, E. Togan, Y. Chu, A.S. Trifonov, E. Kaxiras, and M. D. Lukin, New J. Phys. 13, 025025 (2011).

[32] A. E. Hughes and W. A. Runciman, Proc. Phys. Soc. London 90, 827 (1967).

[33] G. Davies and M.F. Hamer, Proc. R. Soc. A 348, 285 (1976).

[34] E. van Oort and M. Glasbeek, Chem. Phys. Lett. 168, 529 (1990).

[35] F. Dolde, H. Fedder, M. Doherty, T. Nöbauer, F. Rempp, G. Balasubramanian, T. Wolf, F. Reinhard, L. Hollenberg, F. Jelezko,, et al., Nature Phys. 7, 459 (2011).

[36] A. Stacey, T. J. Karle, L. P. McGuinness, B. C. Gibson, K. Ganesan, S. Tomljenovic-Hanic, A.D. Greentree, A. Hoffman, R. G. Beausoleil, and S. Prawer, Appl. Phys. Lett. 100, 071902 (2012).

[37] A. Stacey, D. A. Simpson, T. J. Karle, B. C. Gibson, V. Acosta, Z. Huang, K-M. C. Fu, C. Santori, R. G. Beausoleil, L.P. McGuinness, K. Ganesan, S. Tomljenovic-Hanic, A.D. Greentree, and S. Prawer [Adv. Mater. (to be published)].

[38] See Supplemental Material at http://link.aps.org/ supplemental/10.1103/PhysRevLett.108.206401 for details. 
[39] K.-M. C. Fu, C. Santori, P. E. Barclay, L. J. Rogers, N. B. Manson, and R.G. Beausoleil, Phys. Rev. Lett. 103, 256404 (2009).

[40] P. Tamarat, N. B. Manson, J. P. Harrison, R. L. McMurtrie, A. Nizovtsev, C. Santori, R. G. Beausoleil, P. Neumann, T. Gaebel, F. Jelezko, P. Hemmer, and J. Wrachtrup, New J. Phys. 10, 045004 (2008).

[41] A. Batalov, V. Jacques, F. Kaiser, P. Siyushev, P. Neumann, L. J. Rogers, R. L. McMurtrie, N. B. Manson, F. Jelezko, and J. Wrachtrup, Phys. Rev. Lett. 102, 195506 (2009).

[42] C. Santori, D. Fattal, S. M. Spillane, M. Fiorentino, R. G. Beausoleil, A. D. Greentree, P. Olivero, M. Draganski, J. R. Rabeau, P. Reichart, B. C. Gibson, S. Rubanov, D. N. Jamieson, and S. Prawer, Opt. Express 14, 7986 (2006).

[43] C. Santori, P. Tamarat, P. Neumann, J. Wrachtrup, D. Fattal, R. G. Beausoleil, J. Rabeau, P. Olivero, A. D. Greentree, S. Prawer, F. Jelezko, and P. Hemmer, Phys. Rev. Lett. 97, 247401 (2006)
[44] V. M. Acosta, A. Jarmola, E. Bauch, and D. Budker, Phys. Rev. B 82, 201202 (2010).

[45] F. Jelezko, I. Popa, A. Gruber, C. Tietz, J. Wrachtrup, A. Nizovtsev, and S. Kilin, Appl. Phys. Lett. 81, 2160 (2002).

[46] A. Drabenstedt, L. Fleury, C. Tietz, F. Jelezko, S. Kilin, A. Nizovtzev, and J. Wrachtrup, Phys. Rev. B 60, 11503 (1999).

[47] G. Waldherr, J. Beck, M. Steiner, P. Neumann, A. Gali, T. Frauenheim, F. Jelezko, and J. Wrachtrup, Phys. Rev. Lett. 106, 157601 (2011).

[48] J. P. Hadden, J.P. Harrison, A. C. Stanley-Clarke, L. Marseglia, Y.L. D. Ho, B. R. Patton, J.L. O'Brien, and J. G. Rarity, Appl. Phys. Lett. 97, 241901 (2010).

[49] B. J. M. Hausmann, B. Shields, Q. Quan, P. Maletinsky, M. McCutcheon, J. T. Choy, T. M. Babinec, A. Kubanek, A. Yacoby, M. D. Lukin, and M. Loncar, Nano Lett. 12, 1578 (2012).

[50] A. Faraon, C. Santori, Z. Huang, V. M. Acosta, and R. G. Beausoleil, arXiv:1202.0806. 